\title{
Central Nervous System Solitary Fibrous Tumor/Hemangiopericytoma, Grade 3
}

National Cancer Institute

\section{Source}

National Cancer Institute. Central Nervous System Solitary Fibrous

Tumor/Hemangiopericytoma, Grade 3. NCI Thesaurus. Code C129527.

A solitary fibrous tumor/hemangiopericytoma that arises from the central nervous system. It most often corresponds to the tumor previously diagnosed as anaplastic hemangiopericytoma. 